\title{
Mesenchymal stem cells. A potential source for skeletal repair
}

W E Fibbe

Ann Rheum Dis 2002;61 (Suppl II):ii29-ii3 1

$\mathrm{T}$ he bone marrow serves as a reservoir for different classes of stem cells. In addtion to haematopoietic stem cells the bone marrow comprises a population of marrow stromal cells or mesenchymal stem cells (MSCs). Stromal cells exhibit multilineage differentiation capacity, and are able to generate progenitors with restricted developmental potential, including fibroblasts, osteoblasts, adipocytes, and chondrocyte progenitors. ${ }^{1-3}$

Recently, techniques have become available to isolate and grow mesenchymal progenitors and to manipulate their growth under defined in vitro culture conditions. As a result MSCs can be rapidly expanded to numbers that are required for clinical application. Here the role of MSCs for repair of bone and cartilage is discussed.

\section{MARROW STROMAL CELLS}

Marrow stromal cells comprise a heterogenous population of cells, including reticular endothelial cells, fibroblasts, adipocytes, and osteogenic precursor cells that provide growth factors, cell to cell interactions, and matrix proteins that play a part in the regulation of haematopoiesis..$^{4-6}$ The notion that a stromal microenvironment could support haematopoiesis followed by the development of the long term bone marrow culture by Dexter. In this system an adherent bone marrow derived stromal culture could support the production of haematopoietic progenitor cells over a period of several weeks to months. Friedenstein ${ }^{7}$ already described a population of adherent cells from the bone marrow that were non-phagocytic and exhibited a fibroblast-like appearance. Upon culture at low density either as whole bone marrow or after separation over a density gradient the cells formed characteristic colonies derived from a single precursor, referred to as colony forming unit fibroblastic or CFU-F. After ectopic transplantation under the kidney capsule these cells gave rise to a broad spectrum of differentiated connective tissues including bone, cartilage, adipose tissue, and myelosupportive stroma. ${ }^{78}$ Based on these observations it was proposed that these tissues were derived from a common precursor cell residing in the bone marrow, termed the stromal stem cell, the bone marrow stromal stem cell, the mesenchymal stem cell, or the skeletal stem cell. ${ }^{10}$

MSCs are present in post-natal bone marrow and also in the bone marrow of adults and there is evidence that the frequency declines with age. ${ }^{1}$ For instance the frequency in the newborn is about $1 / 10,{ }^{4}$ which decreases to about $1 / 2 \times 10^{6}$ in an 80 years old person. After allogeneic stem cell transplantation the frequency of CFU-F is transiently reduced in children and recovers over a period of several years, while the defect is permanent in adult recipients of stem cell grafts. ${ }^{11}$ These observations formed the basis for the clinical application of culture expanded MSCs in the context of allogeneic stem cell transplantation.

\section{SOURCES AND PHENOTYPE OF MESENCHYMAL} STEM CELLS

Althought the bone marrow serves as the primary reservoir for MSCs, there presence has been reported in a variety of other tissues. These include periosteum and muscle connective tissue, ${ }^{12}{ }^{13}$ fetal bone marrow, liver, blood. ${ }^{14}$ It is still an ongoing debate as to their presence in steady state peripheral blood, their frequency probably being extremely low. ${ }^{15}$ MSCs have been identified in cytokine (G-CSF) mobilised peripheral blood by some investigators, ${ }^{16}$ although other studies were negative. ${ }^{17}$ MSCs have been identified in fetal blood ${ }^{14}$ and by some laboratories also in umbilical cord blood. ${ }^{18} 19$ However, other laboratories have been unabe to grow MSCs from umbilical cord blood.$^{20}$ The frequency of MSCs in these sources is very low. In fetal blood the frequency has been reported to decline with gestational age, from about $1 / 10^{6}$ mononuclear cells in first trimester fetal blood to $0.3 / 10^{6} \mathrm{MNC}$ in term cord blood. ${ }^{14}$

At present no unique phenotype has been identified that permits the reproducible isolation of MSC precursors with predictable developmental potential. The isolation and characterisation of stromal cell function therefore still relies primarily on their ability to adhere to plastic and their expansion potential. Standard conditions for expansion of MSCs include the presence of serum, in most instances fetal bovine serum. Cell density is a critical factor affecting the growth of cells. Culture attemps are usually unsuccesfull below a critical cell density. The cells can be grown directly-that is, unmanipulated after collection-or after density gradient separation. The CFU-F limiting dilution assay has been used to determine the frequency in bone marow.

A number of markers are expressed on MSCs and some of these have been used to enrich MSCs from populations of adherent bone marrow stromal cells. For instance, CFU-F enrichment has been accomplished using the STRO-1 antibody. ${ }^{21}$ The CFU-F precursor cell was in the CD34 ${ }^{\text {neglow }}$, $\mathrm{CD}^{2} 5^{\text {neg }}$, glycophorin- $\mathrm{A}^{\text {neg }}$ fraction and expressed the markers Thy-1 (CD90), CD106 (VCAM), the $\beta$-1 integrin CD29/CD49, as well as CD10, CD13, and receptors for PDGF, EGF, NGF, and IGFI.

The immune-phenotypical characterisation is usually applied on culture expanded cells and not on primary cells. Characteristic markers for expanded MSCs have been reported and designated SH-2, SH-3, and SH-4. The SH-2 antibody raised against human bone marrow derived MSCs recognises an epitope of endoglin (CD 105), the TGF $\beta$ receptor III present on endothelial cells, syncytiotrophoblasts, macrophages, and connective tissue stromal cells. ${ }^{22}$ The $\mathrm{SH}-3$ and SH-4 antibodies recognise epitopes on human MSCs, the antigens now being identified as distinct epitopes of CD73, an antigen also involved in the activation of B lymphocytes. ${ }^{23}$ However, none of these markers are specific for MSCs, thus hampering the isolation of pure populations of MSCs. In addition, expanded MSCs express HLA class-I but not HLA class-II antigens and lack expression of costimulatory molecules.

Abbreviations: MSC, mesenchymal stem cell; CFU-F, colony forming unit fibroblastic 


\section{MULTILINEAGE POTENTIAL OF MESENCHYMAL STEM CELLS}

The differentiation potential of MSCs into multiple mesenchymal lineages - that is, bone, cartilage and adipose tissue-is most commonly used as a functional criterium defining MSC precursor cells. After culture expansion in vitro human MSC exhibit a spindle shape fibroblastic morphology. It has been reported that a proportion of the initial adherent bone marrow derived stromal colonies are multipotent and maintain multilineage potential into osteogenic, condrogenic, and adipogenic lineages. ${ }^{2}$ These results suggest that the progeny of MSC after culture expansion retain multipotentiality. Human MSC derived from bone marrow have been reported to maintain their differentiation capacity into the osteogenic lineages for over 40 cell doublings. ${ }^{24}$

To promote adipogenic differentiation expanded MSCs are cultured in the presence of dexamethason, methylisobutylxanthine, insulin, and indomethacin. ${ }^{2}$ After induction cells accumulate lipid rich vacuoles that can be stained with Oil Red O. MSCs can also be promoted to differentiate into the chondrogenic lineage when cultured without serum in the presence of transforming growth factor. ${ }^{25}$ Cells can be stained for type 2 collagen, which is characteristic for articular cartilage.

When cultured in the presence of dexamethason and ascorbic acid, purified MSCs undergo a development characterised by the transient induction of alkaline phosphatase, expression of bone matrix protein mRNAses, and deposition of calcium. ${ }^{2}$ In the CFU-F progeny, a proportion seems to exhibit osteogenic potential and become alkaline phosphatase positive. These colonies are able to form foci of mineralised bone and therefore contain osteoprogenitor cells. ${ }^{26}$

\section{(PRE)CLINICAL RESULTS OF MESENCHYMAL STEM CELL TREATMENT}

Based on the in vitro observations to differentiate into the osteogenic and chondrogenic lineage, attemps have been undertaken to use expanded MSCs for in vivo tissue repair. A number of studies have reported the in vivo osteogenic potential of bone marrow derived stromal cells. Bone marrow derived MSCs have been loaded on extracellular matrices such as hydroxyapetite-tricalcium phosphate. After in vivo implantation into NOD/SCID mice bone formation was observed. ${ }^{27}$ In various animal models MSCs have been used for repair of critical size segmental bone defects. For instance, HA/TCP matrices loaded with MSCs have been applied into a femoral gap model in canines. ${ }^{28}$ At three to four months after implantation new bone formation was observed. However, in this model no continguous bone formation occurs and therefore other matrices such as partially deminerised bone may be more usefull. Similar sudies have been performed in baboons using allogeneic MSCs. In these studies no antibody production was observed suggesting that no immune rejection had occurred and that allogeneic MSCs may be used rather than autologous bone marrow derived MSCs.

Pereira $e a^{29}$ infused normal mouse bone marrow derived stromal cells into irradiated transgenic recipient mice with an osteogenesis imperfecta phenotype. Several months after transplantation they demonstrated the presence of donor derived MSCs into various organs of recipient mice, including bone, cartilage, lung, and spleen. MSCs that home to the bones, differentiated into osteocytes and produced normal levels of collagen type I with partial restoration of the osteogenesis imperfecta phenotype. Similar results have been obtained after allogeneic bone marrow transplantation in patients with osteogenesis imperfecta. ${ }^{30}{ }^{31}$ About $2 \%$ of the osteoblasts were of donor origin, suggesting that donor derived MSC precursors in the marrow were capable of homing to the bone marrow and differentiation into osteoblasts or that residual osteoblast in the bone marrow graft were able to engraft in the bone marrow. ${ }^{30}$ In three of five patients with verified osteoblast engraftment after transplantation, an increase in bone mineral content and in body length was observed in comparison with age matched controls. ${ }^{31}$ In these studies osteoblast engraftment may have resulted from the passive transfer of residual osteoblasts in the marrow graft. These data indicate that osteoblasts may be newly formed or may be transplanted successfully in patients with osteogenesis imperfecta. However, it remains to be determined to what extent the cells contributed to the clinical improvement reported for these patients.

MSCs have also been used for in vivo cartilage formation in animal models. ${ }^{32-34}$ Allogeneic bone marrow derived goat MSCs were injected in combination with a hyaluronan carrier into goat knees at several weeks after medial meniscectomy and resection of the anterior crucial ligament. ${ }^{32}$ Regeneration of meniscal tissue was observed in the majority of goats and in comparison with hyaloronan treated controls, less cartilage destruction was observed with preservation of the articular lining. Labelling of the cells indicated the donor origin of the regenerated cartilage. MSCs treated animals exhibited also less bone resorption, subchondral bone remodelling, and osteophyte formation. Tissue biopsy specimens did not show signs of inflammation, suggesting that no immune rejection had occurred. In canines, full repair was observed after intraarticular injection of autologous MSCs of large full thickness defects of the weight bearing region of the articular cartilage. ${ }^{34}$

Recently, autologous culture expanded bone marrow derived MSCs have been applied in patients with osteoarthritis. ${ }^{34}$ Twelve patients received MSC treatment by injection of the cells into the articular cartilage defect of the medial femoral condyle of the knee joint at the time of high tibial osteotmy. The injected MSCs were covered with autologous periosteum. Twelve other patients undergoing the same procedure served as controls and received no cells. In comparison with the control group, patients treated with MSCs had a better arthroscopic and histological grading score. Although the clinical improvement was not significantly different between the two groups, these studies illustrate the feasibility and potential of MSC therapy for cartilage repair. ${ }^{34}$

\section{CONCLUSION}

MSCs transferred in a bone marrow graft may play a potential therapeutic part in the correction of osteogenesis imperfecta. However, controlled studies are required to further substantiate this effect. Preclinical work clearly shows the potential of local MSC treatment for bone and cartilage repair and clinical studies in a variety of conditions are being started. MSCs may also serve as vehicles for gene therapy. For instance, the in vivo osteogenic activity of an adenovirus expressing bone morphogenetic protein 7 has been shown in mice, suggesting that this may be a viable tool to promote bone regeneration in vivo. ${ }^{35}$

A number of fundamental questions relating to the biology of MSCs are still unanswered. To further advance bone and cartilage tissue engineering, a better understanding of progenitor cells and the role of their microenvironment in osteogenic and chondrogenic differentiation and development is required. What is the relation between MSC immunephenotype and function? Are MSCs derived from different sources functionally similar and do culture expanded cells retain their self renewal and multilineage differentiation potential? Continued fundamental and clinical studies are required to turn these developments into a therapeutic reality.

Author's affiliations

W E Fibbe, Laboratory of Experimental Haematology, Department of Haematology, Leiden University Medical Centre, Leiden, Netherlands Correspondence to: Dr W E Fibbe, Department of Haematology, Leiden University Medical Centre, Location C2R - Room 137, 2333 AA Leiden, Netherlands; W.E.Fibbe@LUMC.nl 


\section{REFERENCES}

1 Caplan Al. The mesengenic process. Clin Plast Surg 1994;21:429-35.

2 Pittenger MF, Mackay AM, Beck SC, et al. Multilineage potential of adult human mesenchymal stem cells. Science 1999;284:143-7.

3 Deans RJ, Moseley AB. Mesenchymal stem cells: biology and potential clinical uses. Exp Hematol 2000; 28:875-84.

4 Tavassoli M, Friedenstein A. Hemopoietic stromal microenvironment. Am J Hematol 1983; 15:195-203.

5 Lichtman MA. The ultrastructure of the hemopoietic environment of the marrow: a review. Exp Hematol 1981;9:391-410.

6 Allen TD, Dexter TM, Simmons PJ. Marrow biology and stem cells. Immunol Ser 1990;49:1-38.

7 Friedenstein AJ, Deriglasova UF, Kulagina NN, et al. Precursors for fibroblasts in different populations of hematopoietic cells as detected by the in vitro colony assay method. Exp Hematol 1974;2:83-92.

8 Owen M. Marrow stromal stem cells. J Cell Sci Suppl 1988;10:63-76.

9 Prockop DJ. Marrow stromal cells as stem cells for nonhematopoietic tissues. Science 1997;276:71-4.

10 Gerson SL. Mesenchymal stem cells: no longer second class marrow citizens. Nat Med 1999;5:262-4.

11 Galotto M, Berisso G, Delfino L, et al. Stromal damage as consequence of high-dose chemo/radiotherapy in bone marrow transplant recipients. Exp Hematol 1999;27:1460-6.

12 Nathanson MA. Bone matrix-directed chondrogenesis of muscle in vitro. Clin Orthop 1985;200:142-58.

13 Nakahara H, Dennis JE, Bruder SP, et al. In vitro differentiation of bone and hypertrophic cartilage from periosteal-derived cells. Exp Cell Res 1991; 195:492-503.

14 Campagnoli C, Roberts IA, Kumar S, et al. Identification of mesenchymal stem/progenitor cells in human first-trimester fetal blood, liver, and bone marrow. Blood 2001;98:2396-402

15 Zvaifler NJ, Marinova-Mutafchieva L, Adams G, et al. Mesenchymal precursor cells in the blood of normal individuals. Arthritis Res 2000;2:477-88

16 Fernandez M, Simon V, Herrera G, et al. Detection of stromal cells in peripheral blood progenitor cell collections from breast cancer patients. Bone Marrow Transplant 1997;20:265-71

17 Lazarus HM, Haynesworth SE, Gerson SL, Rosenthal NS, Caplan AI. Ex vivo expansion and subsequent infusion of human bone marrow-derived stromal progenitor cells (mesenchymal progenitor cells): implications for therapeutic use. Bone Marrow Transplant 1995; 16:557-64.

18 Erices A Conget $\mathrm{P}$, Minguell JJ. Mesenchymal progenitor cells in human umbilical cord blood. Br J Haematol 2000;109:235-42.

19 Gutierrez-Rodriguez M, Reyes-Maldonado E, Mayani H. Characterization of the adherent cells developed in Dexter-type long-term cultures from human umbilical cord blood. Stem Cells 2000; 18:46-52.
20 Mareschi K, Biasin E, Piacibello W, et al. Isolation of human mesenchymal stem cells: bone marrow versus umbilical cord blood. Haematologica 2001;86:1099-100.

21 Gronthos S, Simmons PJ. The biology and application of human bone marrow stromal cell precursors. J Hematother 1996;5:15-23.

22 Barry FP, Boynton RE, Haynesworth S, Murphy JM, Zaia J. The monoclonal antibody $\mathrm{SH}-2$, raised against human mesenchymal stem cells, recognizes an epitope on endoglin (CD 105). Biochem Biophys Res Commun 1999;265: 134-9

23 Barry FP, Boynton R, Murphy M, Zaia J. The SH-3 and SH-4 antibodies recognize distinct epitopes on CD73 from human mesenchymal stem cells. Biochem Biophys Res Commun $2001 ; 289: 519-24$.

24 Reyes $M$, Lund $T$, Lenvik $T$, et al. Purification and ex vivo expansion of postnatal human marrow mesodermal progenitor cells. Blood postnatal human marc

25 Mackay AM, Beck SC, Murphy JM, et al. Chondrogenic differentiation of cultured human mesenchymal stem cells from marrow. Tissue Eng 1998;4:415-28

26 Aubin JE. Bone stem cells. J Cell Biochem Suppl 1998;30-31:73-82.

27 Krebsbach PH, Kuznetsov SA, Satomura K, et al. Bone formation in vivo: comparison of osteogenesis by transplanted mouse and human marrow stromal fibroblasts. Transplantation 1997;63:1059-69.

28 Livingston T, Kadiyali S, Elkalay M, et al. Repair of canine segmental bone defects using allogeneic mesenchymal stem cells. Transactions of of the 47th Annual ORS meeting 2001:26:49.

29 Pereira RF, O'Hara MD, Laptev AV, et al. Marrow stromal cells as a source of progenitor cells for nonhematopoietic tissues in transgenic mice with a phenotype of osteogenesis imperfecta. Proc Natl Acad Sci USA 1998;95: $1142-7$

30 Horwitz EM, Prockop DJ, Fitzpatrick LA, et al. Transplantability and therapeutic effects of bone marrow-derived mesenchymal cells in children with osteogenesis imperfecta. Nat Med 1999:5:309-13.

31 Horwitz EM, Prockop DJ, Gordon PL, et al. Clinical responses to bone marrow transplantation in children with severe osteogenesis imperfecta. Blood 2001;97:1227-3.

32 Murphy JM, Kavalkovitch KW, Fink D, Barry FP. Regeneration of meniscal tissue and protection of articulat cartilage by onjection of mesenchymal stem cells. Osteoarthritis Cartilage 2000;8 (suppl B):S25.

33 Wakitani S, Yamamoto T. Response of the donor and recipient cells in mesenchymal cell transplantation to cartilage defect. Microsc Res Tech 2002;58:14-18.

34 Wakitani S, Imoto K, Yamamoto T, Saito M, Murata N, Yoneda M Human autologous culture expanded bone marrow mesenchymal cell transplantation for repair of cartilage defects in osteoarthritic knees. Osteoarthritis Cartilage 2002;10:199-206.

35 Franceschi RT, Wang D, Krebsbach PH, Rutherford RB. Gene therapy for bone formation: in vitro and in vivo osteogenic activity of an adenovirus expressing BMP7. J Cell Biochem 2000;78:476-86 